\title{
Making the News Interesting*
}

\section{Understanding the Relationship Between Familiarity and Interest}

\author{
Frans van der Sluis \\ Human Media Interaction \\ University of Twente \\ Enschede, P.O. Box 217 \\ The Netherlands \\ f.vandersluis@utwente.nl
}

\author{
Richard J. Glassey \\ School of Computing \\ Robert Gordon University \\ Aberdeen, AB25 1HG \\ Scotland \\ r.j.glassey@rgu.ac.uk
}

\author{
Egon L. van den Broek \\ Media and Network Services \\ TNO Technical Sciences \\ Delft, P.O. Box 5050 \\ The Netherlands \\ vandenbroek@acm.org
}

\begin{abstract}
News feeds are an important element of information encountering, feeding our (new) interests but also leading to a state of information overload. Current solutions often select information similar to the user's interests. However, long-term interest in one topic, and being highly familiar with that topic, does not necessarily imply an actual interest response will occur when more of the same topic is selected. This study explores how important familiarity is in predicting an interest response. In a study with 30 subjects, interest was manipulated by topical familiarity using novel stimuli from a popular news source. This study shows, within this context, familiarity is moderately important for an interest response: familiarity does indeed make the news interesting, but only to a certain extent. The results set a baseline for predicting interest during information encountering, indicating familiarity is important, but not the only influential variable a system should consider when selecting information for users.
\end{abstract}

\section{Categories and Subject Descriptors}

H.3.3 [Information Storage and Retrieval]: Information Search and Retrieval-information filtering; H.1.2 [Information Systems]: User/Machine Systems - human information processing

\section{General Terms}

Human Factors, Experimentation, Performance

\begin{abstract}
*ACM, 2012. This is the authors version of the work. It is posted here by permission of ACM for your personal use. Not for redistribution. The definitive version was published in Sluis, F. van der, Glassey, R.J., \& Broek, E.L. van den (2012). Making the news interesting: Understanding the relationship between familiarity and interest. In J. Kamps, W. Kraaij, and N. Fuhr (Eds.), ACM Proceedings of the 4th Information Interaction in Context Symposium (IIiX'12), p. 314-317. August 21-24, Nijmegen, The Netherlands. http://doi.acm.org/10.1145/2362724.2362783.
\end{abstract}

Permission to make digital or hard copies of all or part of this work for personal or classroom use is granted without fee provided that copies are not made or distributed for profit or commercial advantage and that copies bear this notice and the full citation on the first page. To copy otherwise, to republish, to post on servers or to redistribute to lists, requires prior specific permission and/or a fee.

IIiX 2012, Nijmegen, The Netherlands

Copyright 2012 ACM 978-1-4503-1282-0/2012/08 ...\$15.00.

\section{Keywords}

Interest, Familiarity, Information Feeds, Filtering and Recommender Systems

\section{INTRODUCTION}

News feeds have become an important element within our information environment. They provide a stream of novel information that keeps us informed, and pervade many aspects of our life from casual social news feeds that let us know the minutiae of our friends lives, to the important business news feeds that keep us informed of upcoming opportunities and challenges. Although news feeds can greatly augment our personal information horizon (Sonnenwald, 1999) and provide a positive information encountering experience, the reality can often be a source of challenges. Denning (2006) describes the current flow of information as experienced by a user as "infoglut", where increasing amounts of cheap information reach a user and lead to a state of information overload. Despite the benefits of news feeds, the capacity of a person to deal with increasing amounts of information effectively is becoming overstretched.

Automatic solutions have been developed to improve the flow of information by filtering and recommending information based on more sophisticated representations of user interests. Most filtering and recommender systems are based on the assumption that information similar to previously selected information, or matching similar users, will lead to better information streams and an increased interest response (cf. Konstan and Riedl, 2012). For example, Billsus and Pazzani (2007) describe an adaptive news system that provides a higher ranking to news items that are closer to the interests of a user. The interests were derived from previous interactions with the system. For the adaptive system, stories were selected from an average higher rank as compared to a non-adaptive baseline system, indicating a positive influence of familiarity (Billsus and Pazzani, 2007). Although a positive influence of familiarity on interest is intuitive and supported by, at least, indirect evidence, the extent and strength of this influence for filtering and recommending news is difficult to pinpoint.

Despite these advances, challenges remain with understanding and modeling user interest. One potential area of opportunity is to re-evaluate user interest in terms of an emotional experience. Glassey and Azzopardi (2011) describes the Interest Machine (IM), a hypothetical machine aware of our "emotional experience of interest" (Silvia, 2001), that aims to give valued information at the right time. The 
IM learns from the responses (e.g., physiological, interaction activity) of a user, acknowledging the importance of previous and current states (e.g. boredom) of the user in identifying a causal relationship between new information and an interest response. A key challenge besides learning such relationships is applying them appropriately to ensure the flow of information provides a satisfying experience in terms of focal and peripheral interests, without blocking information that could serendipitously lead to new interests. This leads to a general challenge where a system is aware of the affective responses to media and uses this awareness to predict and improve the user experience (Van den Broek et al., 2010).

However, 'interest' is a complicated concept. It differs from long-term interests (n.b. plural); interests do not necessarily lead to an emotional experience of interest, and interest often does not guarantee (but is a requirement for) the development of interests (Silvia, 2001). In other words, having a long-term interest in a certain topic (and, hence, being highly familiar with the topic) does not imply an automatic interest response. The relation between topical familiarity and interest is not unequivocal, with studies showing either positive, neutral, or negative relations (for an overview, see Schraw and Lehman, 2001). Two effects explain these differences. Firstly, when the reader lacks the required background knowledge for a text, the reader will appraise the comprehensibility negatively. On the contrary, texts that are informationally complete do not require the reader to be familiar with the topic (Wade et al., 1999), indicating topical familiarity is only significant when the text is not explicatory. Secondly, Kintsch (1980) proposed an inverted-U relation between background knowledge and interest, where exceptionally low and high familiarity diminish an interest response. This can be interpreted by the novelty of information, being either too novel or too familiar.

When applying the outlined effects of familiarity to a news feed, the context is delineated. A news feed can be expected to not be explicative; without appropriate background knowledge a news item will loose its value, making topical familiarity important to comprehend the news item. Additionally, a news feed is intended to deliver recent content. Although not necessarily so, recent items are likely novel to the reader (Barry, 1994; Xu and Chen, 2006) and, accordingly, unlikely to be very familiar to the reader. Controlling for both the novelty (recency) and complexity (nonexplicatory) of the presented items, this study will investigate the importance of familiarity on creating an interest response.

Interest will be manipulated through sorting out texts which are either of a familiar topic or an unfamiliar topic. It is expected that familiarity will allow readers to make sense of and value a news item, being decisive for the experience of interest. Section 2 will describe the set of articles and experimental setup in detail. The results of the familiarity manipulation will be reported in Section 3. Section 4 will discuss the results, in particular reflecting on how important familiarity is for the emotional experience of interest.

\section{METHOD}

\subsection{Participants}

In total $N=30$ participants voluntarely joined in the experiment, mean age 28.60 years $(S D=6.06)$. None of the participants were native speakers, but all graded their reading literacy highly $(M=4.63 ; S D=.62$; range 1 to 5 , 5 highest).

\subsection{Materials}

\subsubsection{Dataset}

As dataset, 18 articles were selected from The Guardian news feeds ${ }^{1}$. Table 1 describes the selection in terms of the news feeds used as source. The selected news items differed in topic to assure a variation in topical familiarity would be found, and only background articles giving a thorough examination of a topic were selected to enlarge the influence of topical familiarity. The most suitable articles were found in the science feed (See Table 1).

To confound any effects of article length, all articles were truncated after 1200 characters. The cut-off point was placed before the end of the word at position 1200 and three dots were added to indicate the story normally would continue. Any layout was stripped from the articles, leaving only the title and main content to be presented.

\subsubsection{Topical Familiarity Questionnaire}

The subject's familiarity with the main topics of each of the selected articles was rated using a 7-point Likert scale. Familiarity ratings are commonly used to indicate the memory strength for a topic (Wixted and Stretch, 2004). As topics the keywords supplied by The Guardian were used. These keywords represent the topics on a general level (e.g., viruses) as well as a concrete level (e.g., bird flu).

For each article, the three most specific keywords were presented to the participant, where more specific keywords can be expected to give a more fine-grained indication of the knowledge of the subject. When articles had overlapping keywords, the keywords were only presented once. The resulting scores were averaged per article and will henceforth be referred to as topical familiarity.

\subsubsection{Article Questionnaire}

The article questionnaire was presented after the subject read an article. In accordance with related studies (e.g., Silvia, 2006, 2008), interest was measured using two differentials: interesting-uninteresting and boring-exciting (Silvia, 2008). Furthermore, a Likert scale was added specifically to benefit from the shortened texts (See Section 2.2.1), asking the participant to agree with the statement "I would be interested in reading more of this text". All three questions

$1_{\text {www.guardian.co.uk }}$

Table 1: Selection of articles specified by originating feed.

\begin{tabular}{lr}
\hline Feed & Items \\
\hline Comment is free & 2 \\
Culture & 1 \\
Environment & 2 \\
Latest financial, market, and economic & 1 \\
Life and style & 2 \\
Science & 8 \\
Technology & 2 \\
\hline
\end{tabular}




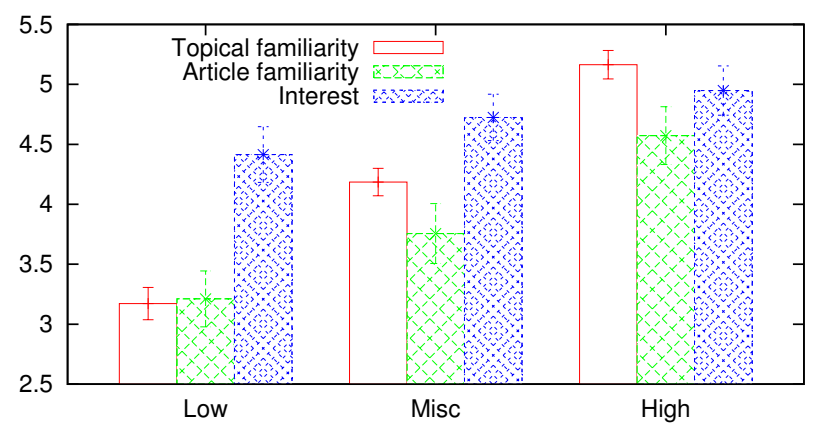

Figure 1: Comparison of interest, topical familiarity, and article familiarity by experimental condition showing the means and confidence intervals.

formed a reliable scale, confirmed by an excellent Cronbach's alpha of .92 $(N=540)$. Only the scale (i.e., the average of the three items) will be reported furtheron.

In order to confirm the manipulation of familiarity, a semantic differential was added to the article questionnaire, inquiring whether the article was familiar-unfamiliar. This scale will henceforth be referred to as article familiarity, highlighting the difference between actual familiarity with the article and expected familiarity via the topics of the article (See Section 2.2.2). All answer possibilities were on a 7-point scale.

\subsection{Design and Procedure}

A within-subjects design was used, showing all articles to each participant. For each participant, the articles were grouped based on the average topical familiarity score per article. Three blocks were created based on this score, containing the six highest, six lowest, and six miscellaneous articles. The order of the blocks was counter-balanced, giving a total of $3 !(=6)$ conditions.

The experiment started with a few general questionnaires about demographics and curiosity, followed by the topical familiarity questionnaire. Then, grouped per block, each of the articles was shown for self-paced reading, after which the participant continued to the article questionnaire. After each block a questionnaire about the past experience was given. Each of the parts was preluded with a short textual instruction. The goal of the experiment was not made explicit and all participants were instructed to read thoroughly though self-paced. The total experiment lasted on average 50 minutes.

\section{RESULTS}

For each of the conditions (i.e., the three levels of topical familiarity), Figure 1 shows the interest, topical familiarity as asked beforehand, and article familiarity as asked after each news item, including the $\alpha=.95$ confidence intervals. The figure shows that each variable differs over the conditions. An analysis of variance confirms this with a significant effect $(p<.05)$ of condition (high, low, and miscellaneous) on the variables: $F(2,537)=31.35, p<.001$ for article familiarity and $F(2,537)=6.28, p<.005$ for interest. The relation between topical familiarity and condition will not be tested, as the conditions were based on the topical familiar- ity scores. The analyses of variance confirm, via the manipulation, the influence of topical familiarity on self-reported article familiarity and interest. Interest and article familiarity were succesfully manipulated by topical familiarity.

The relations between each of the variables is further explored in Table 2, showing the correlations between them. As expected, topical familiarity correlates with article familiarity. This correlation can be interpreted as moderately strong, indicating topical familiarity is only partly capable of predicting actual article familiarity. This highlights the need for sophisticated methods to predict the familiarity a user has with an article.

Table 3 describes a linear regression model $\mathcal{M}$ predicting interest by both types of familiarity: topical and article. The model explains $15.90 \%$ of variance $(F(2,537)=50.91$, $p<.001)$. Having only two, related, variables, this be considered reasonable. Furthermore, the model shows the relative importance of actual article familiarity in comparison to predicted topical familiarity, something also indicated in Table 2 where article familiarity has the highest correlational strength.

The results confirms the expected importance of article familiarity for the occurence of interest within the context of a homogenous set of novel articles taken from a popular news source. Nevertheless, the relatively low strength of the correlations indicates more factors play a role in predicting interest.

\section{DISCUSSION}

This study set out to explore the role of familiarity in predicting an interest response. More generally, the extent to which familiarity changes the value of information. Within a controlled context, where the novelty (recency) and complexity (non-explicatory) of the articles were consistent with what can generally be expected from a news source, article familiarity was found to predict interest with a correlation of $r=.390$. Furthermore, an indication of topical familiarity successfully manipulated $(p<.005)$ and predicted $(r=.219)$ an interest response. Taken together, both article familiarity and topical familiarity explained $15.90 \%$ of variance in interest. This highlights both the pivotal role of familiarity in predicting interest, as well as leaving open an important role for other variables.

This study confirmed an often assumed role of familiarity in contributing to an interest response: familiarity is an important construct in the IM. In particular for news, which consists of novel articles, familiarity explained $15.90 \%$ of variance in interest. However, this also clearly indicates a need for more sophisticated models of interest. As the hypothetical IM postulates, many variables interact in creating an interest response. Aspects of the information, the

Table 2: Correlations between interest, topical familiarity, and article familiarity.

\begin{tabular}{lrrr}
\hline & 1 & \multicolumn{1}{c}{2} & \multicolumn{1}{c}{3} \\
\hline 1. Topical familiarity & 1 & .355 & .219 \\
2. Article familiarity & & 1 & .390 \\
3. Interest & & & 1 \\
\hline Note. All values were significant at $p<.01$.
\end{tabular}


Table 3: Linear regression model $\mathcal{M}$ predicting interest by both topical and article familiarity.

\begin{tabular}{lrrrrc}
\hline & \multicolumn{5}{c}{$\mathcal{M}$} \\
\cline { 2 - 6 } Feature & \multicolumn{1}{c}{$\mathrm{B}$} & $\mathrm{SE}(\mathrm{B})$ & $\beta$ & \multicolumn{1}{c}{$t$} & \multicolumn{1}{c}{$p$} \\
\hline Intercept $\left(B_{0}\right)$ & 3.070 & .221 & & 13.898 & .000 \\
Topical familiarity & .114 & .053 & .092 & 2.172 & .030 \\
Article familiarity & .299 & .035 & .357 & 8.443 & .000 \\
\hline
\end{tabular}

user, and the context can help explain a larger proportion of variance. With a focus on familiarity, this study has set a baseline for future studies to improve on manipulating and explaining interest, including adding more predictors.

The IM not only highlights the complicated mixture of antecedents needed to predict interest, but also the need for direct measurement of interest. Measures such as average ranking precision might be indicative of interest, but leave out a more fine-grained approximation of intensity and persistence of an actual interest response. Aside subjective measures of interest, objective measures such as eye-tracking or psychophysiological sensors can aid in keeping track of the details and intensity of an interest response and more general the affective state of the user. For example, the affective state likely experienced during this study will be profoundly different from a real-life, busy, situation, possibly enlarging the experienced and reported interest.

The findings of this study can be interpreted using the contemporary appraisal theory of interest, which embeds interest within the appraisal theory of emotion, explaining why and predicting when interest will occur by two consecutive appraisals: novelty-complexity and comprehensibility (Silvia, 2006). Familiarity can influence both appraisals: the primary, novelty-complexity, as well as the secondary, comprehensibility. Firstly, familiarity determines the novelty of a new piece of information and influences the appraisal of its complexity. Secondly, familiarity affects the coping potential, where more familiar information will likely be appraised as more comprehensible. Such an interpretation of the findings not only structures the results but also indicates other factors potentially of importance for recommender and filtering systems.

Most recommender system currently try to find information similar to the previous interests or knowledge of a user, or information similar users find interesting (Konstan and Riedl, 2012). The findings of this study indicate this method works within a restricted context and with a limited accuracy, leaving open potential areas to gain predictive power. This study has set a baseline for predicting interest by only familiarity. Hence, seen in the light of the hypothetical IM, familiarity is one of its main variables. When more context will be included, the relation between information and interest can be captured accurately, giving a solution to the current quantity of cheap information encountered daily, the so-called "infoglut".

\section{Acknowledgements}

The authors would like to thank the anonymous reviewers for their valuable comments and suggestions to improve the quality of the paper. This work was part of the PuppyIR project, which is supported by a grant of the 7th Framework ICT Programme (FP7-ICT-2007-3) of the European Union, and was supported by the Dutch national program COMMIT (project P4 on Virtual worlds for well-being).

\section{References}

Barry, C. L. (1994). User-defined relevance criteria: An exploratory study. Journal of the American Society for Information Science and Technology, 45(3):149-159.

Billsus, D. and Pazzani, M. (2007). Adaptive news access. In Brusilovsky, P., Kobsa, A., and Nejdl, W., editors, The Adaptive Web: Methods and Strategies of Web Personalization, volume 4321 of Lecture Notes in Computer Science, pages 550-570. Springer, Berlin / Heidelberg.

Denning, P. J. (2006). Infoglut. Communications of the ACM, 49(7):15-19.

Glassey, R. and Azzopardi, L. (2011). Finding interest in the stream. Proceedings of the American Society for Information Science and Technology, 48(1):1-4.

Kintsch, W. (1980). Learning from text, levels of comprehension, or: Why anyone would read a story anyway. Poetics, 9(1-3):87-98.

Konstan, J. and Riedl, J. (2012). Recommender systems: from algorithms to user experience. User Modeling and User-Adapted Interaction, 22(1):101-123.

Schraw, G. and Lehman, S. (2001). Situational interest: A review of the literature and directions for future research. Educational Psychology Review, 13(1):23-52.

Silvia, P. J. (2001). Interest and interests: The psychology of constructive capriciousness. Review of General Psychology, 5(3):270-290.

Silvia, P. J. (2006). Exploring the psychology of interest. Oxford University Press, New York.

Silvia, P. J. (2008). Appraisal components and emotion traits: Examining the appraisal basis of trait curiosity. Cognition \& Emotion, 22(1):94-113.

Sonnenwald, D. H. (1999). Perspectives of human information behaviour: contexts, situations, social networks and information horizons. In Wilson, T. D. and Allen, D. K., editors, Exploring the contexts of information behaviour, pages 176-190. Taylor Graham Publishing, London, UK.

Van den Broek, E. L., Van der Sluis, F., and Schouten, T. E. (2010). User-centered digital preservation of multimedia. ERCIM (European Research Consortium for Informatics and Mathematics) News, 80:45-47.

Wade, S. E., Buxton, W. M., and Kelly, M. (1999). Using think-alouds to examine reader-text interest. Reading Research Quarterly, 34(2):194-216.

Wixted, J. and Stretch, V. (2004). In defense of the signal detection interpretation of remember/know judgments. Psychonomic Bulletin \& Review, 11:616-641.

Xu, Y. C. and Chen, Z. (2006). Relevance judgment: What do information users consider beyond topicality? Journal of the American Society for Information Science and Technology, 57(7):961-973. 